Eva Lia Wyss

\title{
Die Bildung von Akteur-Netzwerken in Liebesbriefen und -botschaften. Ein Brautbrief des letzten Jahrhunderts und amouröse Postings in Facebook im Vergleich
}

\section{Ausgangslage}

Briefliche Netzwerke bilden über die Philologien hinaus für verschiedene Disziplinen eine erkenntnisreiche Quelle, die für die Rekonstruktion unterschiedlicher Aspekte der Werkgenese oder für die Nachzeichnung von Entwicklungsprozessen in wissenschaftlichen Domänen von großem Nutzen sind. Darunter befinden sich bisweilen Liebeskorrespondenzen wie beispielsweise bei Bettine Brentanos und Achim von Arnims Briefwechsel. ${ }^{1}$

Zudem zeigt ein Blick auf vermeintlich intime Korrespondenzen und Briefe des 19. und 20. Jahrhunderts in vielerlei Hinsicht Aspekte einer über das Liebespaar hinausgehenden Netzwerkbildung. Diese Formen der Netzwerkbildung sollen hier untersucht werden. Im Mittelpunkt stehen soll der Vergleich älterer Praktiken der Liebesbriefe im Vergleich mit neueren in sozialen Medien.

Dieser Fokus bedarf einer Netzwerktheorie, die in Kapitel 2 kursorisch betrachtet wird, worauf in Kapitel 3 mit dem Werkzeug der Akteur-Netzwerk-Theorie (ANT) an Beispielen die Frage der Netzwerkbildung verglichen werden soll und in einem Fazit in Kapitel 4 die Frage nach dem Mehrwert der ANT für die Briefforschung zu diskutieren ist.

\section{Die Bildung von Netzwerken durch briefliche Kommunikation}

Neben elektronischen Circuits werden auch soziale, politische oder wirtschaftliche Netze mit dem aus dem Englischen stammenden „Netzwerk“ bezeichnet.

1 Vgl. Moering 2018.

Eva Lia Wyss, Universität Koblenz-Landau

つ Open Access. ( 2021 Eva Lia Wyss, publiziert von De Gruyter (c) BY-NC-ND Dieses Werk ist lizenziert unter der Creative Commons Attribution-Non-Commercial-NoDerivatives 4.0 International Lizenz. https://doi.org/10.1515/9783110712568-018 
Netzwerke werden dabei als eine überlagerte Struktur untersucht, durch die angezeigt wird, dass Relationen oder Beziehungen zwischen Akteur`innen oder Institutionen bestehen, die mit Jansen als „[...] eine abgegrenzte Menge von Knoten oder Elementen und der Menge der zwischen ihnen verlaufenden sogenannten Kanten“ ${ }^{2}$ darstellbar werden. Dabei interessieren die sich im Netzwerk herausbildenden Relationen zwischen den Akteur^innen. Bei einem Fokus auf Briefe oder Postings verschwindet die beziehungsbildende Funktion des Kommunizierens hinter dem Netzwerk, das als Produkt der Relationen im Blick ist. So geraten viele Aspekte der wechselseitigen Verständigung, der Stabilisierung des Netzwerks in den Hintergrund und machen einen produktorientierten Zugang für eine sprachkulturwissenschaftliche Briefanalyse wenig fruchtbar.

Bei einem Interesse an Kommunikation in sozialen Medien scheint Castells Netzwerktheorie günstig, da durch sie ein expliziter Bezug zur Digitalisierung der Gesellschaft möglich wäre. Doch auch Castells definiert ein Netzwerk sehr nah an dem organisationalen Modell, das die konkrete beziehungsbildende Interaktion ausblendet:

A network is a set of interconnected nodes. A node is the point where the curve intersects itself. A network has no center, just nodes. Nodes may be of varying relevance for the network. Nodes increase their importance for the network by absorbing more relevant information, and processing it more efficiently. The relative importance of a node does not stem from its specific features but from its ability to contribute to the network's goals. [...] Nodes only exist and function as components of networks. The network is the unit, not the node. ${ }^{3}$

Latour beschreibt die mit Castells verbundenen Paradigmenwechsel wie folgt:

\begin{abstract}
Die einen sind natürlich die technischen Netzwerke - Elektrizität, Eisenbahn, Kanalisation, Internet und so weiter. Die zweite Bedeutung wird in der Organisationssoziologie verwendet, um einen Unterschied zwischen Organisationen, Märkten und Staaten einzuführen. Hier stellt das Netzwerk eine informelle Weise dar, menschliche Handlungsträger miteinander zu assoziieren. Wenn Castells den Ausdruck verwendet, verschmelzen die beiden Bedeutungen, denn Netzwerk wird nun zu einem privilegierten Organisationsmodus, gerade aufgrund der Erweiterung der Informationstechnologie. ${ }^{4}$
\end{abstract}

Bei der Analyse von brieflichen Netzwerken interessiert die Modellierung des Netzwerks natürlich auch, allerdings hat die Darstellung im Modell eher synoptischen Resultatscharakter. Weit bedeutender ist aus einer kulturlinguistischen Sicht die Entwicklung, der Auf- und Ausbau, die Persistenz eines Netzwerks. Dies leistet

\footnotetext{
2 Jansen 2003, 58.

3 Castells 2004, 3.

4 Latour 2007, 225.
} 
die (radikal) konstruktivistische Akteur-Netzwerk-Theorie, bei der genau dieses Momentum fokussiert wird. Der Prozess der Interaktion der in einem Netzwerk beteiligten Akteur`innen wird in diesem Kontext als eine wechselseitige Interpretation von Äußerungen (als enrolment beispielsweise durch Übersetzung oder Überschreibung) analysiert, wodurch gezeigt werden kann, welche Bedeutung(en) zwischen verschiedenen beteiligten Akteur ${ }^{\star}$ innen in den Interaktionen und Handlungen ausgebildet werden. ${ }^{5}$

Eine der wichtigsten Maximen der ANT ist die gleichwertige symmetrische Behandlung aller am Netzwerk beteiligten Akteur^innen, ob es sich um menschliche oder nichtmenschliche Lebewesen oder um Objekte oder Dinge handelt. Es gelten als Akteur*innen oder Aktant*innen ,alle Entitäten, denen es mehr oder weniger erfolgreich gelingt, eine Welt voller anderer Entitäten mit eigener Geschichte, Identität und Wechselbeziehungen zu definieren und aufzubauen."6 Eine Besonderheit ist die Annahme, dass auch nichtmenschliche Lebewesen und Dinge als Akteur*innen an der multilateralen Aushandlung teilhaben, indem sie eine (netzwerkbezogene) Identität innehaben, die im Prozess der Netzwerkbildung entwickelt, bestimmt und erprobt wird. ${ }^{7}$

Dabei ist die Annahme einer Symmetrie der an der Netzwerkbildung beteiligten Einheiten zentral. Dies wird mit dem Beispiel einer handgeschriebenen Notiz veranschaulicht: „Der Türschließer streikt. Schließen Sie um Gottes willen die Tür!“, ${ }^{8}$ mit dem die Personen auf einen Defekt und auf die deswegen erforderliche Maßnahme hingewiesen werden, die (in Gottes Namen) zu befolgen sei. Ein solcher Vorschlag wird vom Umfeld meist als sinnvoll und nachvollziehbar ohne Widerstand befolgt. Das Beispiel zeigt die „techno-sozialen Verwicklungen“9 als eine „Verschmelzung von industriellen Beziehungen, Religion, Werbung und Technik“. ${ }^{10}$ Besonders deutlich wird damit die vermeintlich klare Trennung in eine (technologische) Welt der (leblosen) Artefakte und eine (soziale) Welt der (intentio-

5 Die grundlegende rekonstruktive Methode bietet im Kontext wissenschaftlicher und technologischer Innovationen die Möglichkeit, die sich dabei manifestierenden vielfältigen Aushandlungsprozesse darzustellen, bei der sich nicht eine Technologie (gegen andere Vorschläge) durchsetzt. Darüber hinaus ist zugleich die Bildung eines Netzwerks beobachtbar, eines Netzwerks, an dem nicht nur menschliche Akteure, sondern auch nichtmenschliche Lebewesen und dingliche Objekte teilhaben. Darüber hinaus kann sie auf Netzwerke jeder Art angewendet werden, wenn es das Ziel ist, ein Netzwerk nicht als Produkt zu analysieren, sondern es im dynamischen Prozess der Entwicklung zu beobachten. (Vgl. Schulz-Schaeffer 2014, 274 u. 277-278.)

6 Callon 1991, 140.

7 Vgl. Callon 1986, 214.

8 Latour 1996, 62.

9 Johnson 1988, 309.

10 Latour 1992, 227. 
nalen) Sozietäten, die nun gemeinsam als Mitwirkende mit in die Netzwerkbildung integriert werden. Dabei wird die Idee der „Vergemeinschaftung“,11 also eine durch menschliche soziale Handlung erreichte Vernetzung (wie sie im anthropologischen Kontext üblich ist), dezidiert von sich gewiesen und überwunden.

Auf das Objekt des ,Türschließers‘ verweist die handgeschriebene ,Notiz‘, die über den Defekt informiert und zu Folgehandlungen auffordert. Dabei werden bereits ausgehandelte Bedeutungen tradiert, die sich im Kontext von Netzwerken bilden.

Aus methodologischer Sicht erfahren im Rahmen der ANT sämtliche beteiligten Akteur ${ }^{\star}$ innen (Schreiber ${ }^{\star}$ in, Leser ${ }^{\star}$ in und auch der Brief als handelndes Objekt) eine Dynamisierung und Aktivierung, die in der tatsächlichen „Prozessierung“ von Bedeutungen in einem Netzwerk geschieht. Die Akteur`innen werden dabei hinsichtlich der Herstellung von Konvergenzen (bzw. Nicht-Konvergenzen) zwischen den Akteur^innen beschrieben, eine Konvergenz, die das Netzwerk mehr und mehr zu einer Irreversibilität führt, also zu einem stabilen Netzwerk, das gegenüber Schwächungsversuchen widerständig bleibt. Der Prozess der Aushandlung und der kooperativen Zuschreibung von Bedeutungen durch symmetrische Akteure wird als enrolment bezeichnet.

Zentral ist die Tatsache, dass diese Netzwerke nicht einzig als soziale ,menschengemachte' Beziehungen erklärt werden, sondern als solche, die sich durch eine Verkettung, Verzahnung und Verquickung von technologischen und sozialen Akteur*innen bilden. ${ }^{12}$ So lässt sich in diesem Beitrag die ANT-Methode sehr gut auf die briefliche Netzwerkbildung übertragen, wodurch nun auch dem Brief eine Agency zugeschrieben wird.

\section{Liebesbrief und -posting - Liebes- und Paarkommunikation mit einer ANT-Perspektive}

Wenn die ANT-Methode aus dem Bereich der Wissenschaftsforschung auf historische und zeitgenössische Schreib-,Technologien` angewendet wird, hat dies einige Konsequenzen: 1. Paarkommunikative Technologien, ob Brief oder Posting, ${ }^{13}$ werden als mehr oder weniger (un-)abhängige Akteure in Prozessen wahrgenommen

11 Weber 1922, § 9.

12 Hausendorf 2009, 11-12.

13 Die hier verwendeten Liebesbriefe und Postings stammen aus dem Liebesbriefarchiv (LBA) beziehungsweise einer Qualifikationsarbeit eines Studierenden (Bittner 2019). Das LBA wächst stetig an und umfasst heute über 22.000 Briefe, die in Zusammenarbeit mit der Technischen Universität 
und auf ihre Funktion und Wirkweise in den Netzwerken untersucht; ${ }^{14}$ 2. wird die Funktionalität eines individuellen Briefs mit anderen Briefen oder anderen konkreten Kommunikationsereignissen verglichen, wodurch 3. der weitere konkrete Kontext beleuchtet wird, in dem Bedeutungen ausgehandelt werden. Dabei werden 4. diese in einem Brief ausgehandelten Bedeutungen explizit gemacht. Auf diese Weise gelingt es, 5. diese Bedeutungen als ko-konstruierte Bedeutungsangebote im Prozess ihrer Aushandlung in den Fokus zu nehmen und insbesondere die Annahme oder Ablehnung solcher Bedeutungsangebote in der Analyse mitzuberücksichtigen.

Auch ein Brief, ein Posting, ein Kommentar oder ein Like werden dabei zu Akteuren in einem Netzwerk. Auf die darin konstruierten Bedeutungen reagieren andere Akteur ${ }^{\star}$ innen, die damit eine Vielfalt an immanenten kulturellen und sozialen Prämissen, milieuspezifische Verhaltensnormen sowie in den Brief eingeschriebene Präsuppositionen und ihre ,Übersetzungen“ thematisieren. Diese Prozesse werden dabei zugänglich und erlauben es, die Netzwerkbildung (deren Aus- und Umbau) zu untersuchen. So werden damit mehrere Perspektiven der individualmedialen personalen Kommunikation, durch die das ,Geschriebene', das ,Gelesene ' und etwaige mit dem Brief zu lösende ,Aufgaben "15 in den netzwerkbildenden Prozess eingebunden werden, gleichwertig nebeneinander gestellt.

Der Perspektivwechsel erlaubt es zudem, den Liebesbrief ${ }^{16}$ oder auch das Liebesposting ${ }^{17}$ weit über textsemantische und textlinguistische Funktionen hinaus als eines der kooperativen, konstruktiven Elemente zu sehen, als ein (Ding-)Akteur, der in der Folge die Verzahnung von epistolarer Technologie und Sozialem, dem schreibenden oder lesenden Menschen, darstellt.

Darmstadt kontinuierlich digitalisiert, transkribiert und annotiert werden. Die Universitätsbibliothek Koblenz-Landau (Campus Koblenz) übernimmt die Langzeitarchivierung der Archivmaterialien. Ausführliche Informationen finden sich auf der Webseite: https://liebesbriefarchiv.wordpress. com/projekt/

14 Latour kritisiert die These, menschliche Gesellschaften würden aus sich heraus soziale Strukturen ausbilden, und weist darauf hin, dass deren Erhaltung ganz offensichtlich nicht auf der beständigen Interaktion ko-präsenter Gesellschaftsmitglieder beruht. Es sei vielmehr davon auszugehen, dass „soziales Leben, wenigstens in seiner menschlichen Form, von irgendetwas anderem als von der sozialen Welt abhängen [muss].“ (vgl. Latour 1996b, 232, zit. nach Schulz-Schäffer 2014, 287.

15 Hausendorf 2000, 211-212.

16 Vgl. zum Liebesbrief Wyss 2008; Stauf u. Paulus 2013, Hübner, Stauf u. Paulus 2020.

17 Vgl. zum Liebesposting in Sozialen Medien (Facebook) Frick 2014; Wyss 2017. 


\subsection{Beispiel 1: Rudolfs und Emmys Liebesbriefkommunikation}

Der Brief von Emmy stammt aus einer Verlobungskorrespondenz ${ }^{18}$ des Liebesbriefarchivs (LBA) von welcher insgesamt 46 Briefe erhalten sind. Es handelt sich um einen Briefwechsel zwischen Rudolf, einem 27 Jahre alten Hannoveraner Industriellensohn, und seiner ungefähr 17-jährigen Verlobten, Emmy, einer Tochter eines bekannten Schriftstellers aus der Bodenseeregion. Der Brief ist eine schriftliche Kommunikationsform, ${ }^{19}$ wodurch die raumzeitliche Kommunikationssituation - wie Ehlich es bezeichnet ${ }^{20}$ - eine Zerdehnung erfährt, die besondere Anforderungen, wie zum Beispiel der Antizipation, der Leserführung, an die Beteiligten stellt. Dabei kommt der Weg des Briefes in Distanz und Dauer zum Ausdruck. Doch zeigt sich, dass der Liebesbrief, der auf der Grundlage des modernen postalischen Adress-Systems verschickt wird, bei dem die Sendung entweder direkt an Adressat*innen ausgehändigt oder in Briefkästen aufgehoben oder /und durch Mittelspersonen an die Adressat*in weitergereicht wird, einen weiteren Unterschied aufweist. Zugleich ist ein Brief auch immer performativ zu denken, also ein „Ereignis \& Objekt“, ${ }^{21}$ das in einer soziologischen Perspektive auch etwa als eine „Gabe“22 gesehen wird, die insbesondere im Privaten aufbewahrt oder auch in einem institutionellen Rahmen wie dem LBA $^{23}$ archiviert wird.

In Emmys Brief wird ein grundlegender Unterschied durch den Zustellort zum Ausdruck gebracht. Bei einer Zustellung eines Briefs an die elterliche Adresse ist davon auszugehen, dass der Brief im Kreis der Familie vorgelesen wird. Sie bezieht deshalb beim Briefeschreiben diese familiale Vorlesepraktik beim Schreiben mit ein. ${ }^{24}$ Der Brief wird dabei als ein materiales Objekt, das geschrieben, gelesen und in manchen Fällen auch vorgelesen wird, in seinen Auswirkungen weitreichender sein, als man es mit der Übermittlung von sprachlich gefassten Informationen erfassen kann.

Mit der ANT wird der einzelne Brief als ein Akteur gesehen, der an der Bildung des Netzwerks um Emmy und Rudolf teilhat. An dem ausgewählten Schreiben ${ }^{25}$ von Emmy wird dies besonders deutlich. Sie beschreibt in diesem Brief an Rudolf

18 Vgl. Wyss 2008, Wyss 2010.

19 Vgl. Ermert 1979, Nickisch 1991, Schuster 2020.

20 Vgl. Ehlich 2014.

21 Bohnenkamp et al. 2008.

22 Vgl. dazu ausführlich Strobel 2020.

23 Vgl. Anm. 14.

24 Vgl. den Handbuchartikel zum Brautbrief von Lach 2020, 517.

25 Liebesbriefarchiv Koblenz, Sign. LB_00088_0002. 
ihre Position und fordert ihn darin auf, auf die Absicherung ihres Verlöbnisses hinzuarbeiten.

In dem Brief wird klar gemacht, dass Emmy und Rudolf sich zu Beginn ihrer Verlobungszeit befinden und damit in einer Situation, die nicht rasch in ein ,sicheres' Verhältnis überführt werden kann. Auch wenn sich die beiden die Ehe versprochen haben - wohl auch mit dem Einverständnis von Emmys Eltern - bedarf dieses Eheversprechen weiterer Klärungen zwischen den Eltern beider Verlobten. Der Brief steht damit am Anfang eines länger dauernden Prozesses der Stabilisierung des Verlöbnisses, woran die Planung der Hochzeit und die Ehevorbereitung geknüpft werden.

In diesem Brief werden vorsichtige Deutungsangebote verwendet, Rudolf gegenüber wird dargestellt, wie sich Emmys Beziehung zu ihm entwickelt und gefestigt hat, bis zu dem Zeitpunkt, an dem sich ihre Gefühle für Rudolf und ihre Entscheidung für eine Ehe geklärt hatten. Die Entscheidung ist zu diesem Zeitpunkt so stark gefestigt, dass Emmy nun beruhigt ihre gemeinsame Zukunft vor Augen sieht. Doch so stark auch ihr Entschluss bekräftigt wird, so offensichtlich ist die Ambivalenz. Denn die Situation ist nicht gesichert, solange die Aussprache zwischen den Eltern der beiden nicht stattgefunden hat.

Zugleich schaut Emmy mit Sorge auf die zu erwartende Reaktion von Rudolfs Eltern auf ihr zweites Schreiben dieser Tage, das sie - zum ersten Mal - an Rudolfs Heimatadresse gerichtet hatte. Sie geht dabei davon aus, dass dieser Brief - obwohl auch er an Rudolf adressiert ist - nicht als privater Brief, sondern als (halb-)öffentlicher vorgelesen werden würde. Sie befürchtet, der Brief an das Elternhaus „könnte zu viel sprechen”, könnte also Dinge andeuten, die unangebracht und indiskret sein würden.

Damit erfolgt mit dem vorliegenden Brief an Rudolf nicht einzig ein kommunikativer Vorgang, sondern der Brief fungiert zugleich als ein Dokument, mit dem Emmy ihr Verlöbnis dokumentiert und zugleich bestätigt, dass es der beiden fester Wunsch ist, eine Ehe einzugehen. Im Brief wird ferner bekräftigt, wie sie im Vertrauen auf Rudolf zu einer inneren Klarheit gefunden hat.

Da dieser Brief nicht nur die aktuelle Situation ihrer Verbindung reflektiert, sondern eine Reflexion über den ans Elternhaus adressierten Brief vornimmt, stellt er ein neues und stärkeres und insbesondere ein intimeres Band zwischen Rudolf und Emmy her.

Gleichzeitig wird in einigen Bemerkungen angedeutet, dass der Erfolg, die Ehe, ein Engagement von beiden benötigt. Im Kontext einer erzählend-plaudernden ${ }^{26}$

26 Mit dem heute nicht mehr gebräuchlichen „Plaudern“ war damals eine an mündliche Kommunikationspraxis angelehnte schriftliche (zerdehnte) Form des lockeren Austausch über allerlei 
Passage, die mit Lehmkuhl als Kaffee-und-Kuchen-Narrativ zu bezeichnen ist, ${ }^{27}$ in der dies und das gefragt wird, Episoden aus dem Alltag aufgegriffen werden, wird ein Bezug zu einer Erzählung aus einem vorhergehenden Brief Rudolfs hergestellt. Dabei plaudert sie davon, dass Rudolf nach seiner Heimkehr bestimmt prüfend von seiner Mutter gemustert werden würde. Hier wird das Beispiel des Appetits erwähnt, da er damit einen guten Eindruck auf seine Mutter machen würde.

Nicht zuletzt gelingt es in diesem Schreiben, die für die Familie unangenehme Situation in Ermatingen zu schildern: das Gerede derer im Dorf, die sich nach ihrer Brautzeit erkundigen; hier geht es weiter um das Unbehagen des Vaters, dem es nicht gefällt, dass die Verlobung noch nicht „offiziell” ist. Abschließend wird die Situation als „unheimlich”, mit einem starken Ausdruck, bewertet.

Insgesamt zeigt dieser Brief einen umfassenden Netzwerk-Kosmos, in dem auch für heutige Leser*innen über die beiden Verlobten hinaus weitere Akteur`innen zwar knapp, aber dennoch anschaulich positioniert werden. Darüber hinaus werden in Bezug auf das Verlöbnis die Dorfgemeinschaft und weitere Personenkreise genannt.

Ebenso zeigt sich die bereits etablierte Netzwerkbildung. Ein Teil des enrolment, die Rollenzuschreibung und die -übernahme, ist durch das Verlöbnis zwischen Emmy und Rudolf bereits erfolgt. Neben Emmys Eltern ist auch die Dorfgemeinschaft schon im Boot. Rudolfs Eltern aber müssen noch überzeugt werden.

Der Brief leistet mit den vielfältigen Bedeutungsangeboten eine Art Übersetzungsvorarbeit. Rudolf dürfte, wenn er die Ehe anstrebt, diese Bedeutungen aufgreifen und würde wohl dabei versuchen, Konvergenzen zu schaffen zwischen den ihm zugänglichen Akteuren, um auf diesem Weg die Irreversibilität des Verlöbnisses und damit den Plan der Eheschließung mehr und mehr umzusetzen.

Ermatingen, den 23. 10.

1903.

Liebster Rudolf!

Eben schlägt es 7 Uhr in der

Dorfkirche - ich denke an Deine

Worte, dort am weißen Horn

als du mich ahnen ließest, daß ich dir mehr sei, als nur eine angenehme Mädchenerscheinung.

Wie pochte mein

Herz mächtig, als mir zum
Bewusstsein kam, du könntest mich wahrhaft lieben. Jener

Moment war wohl der schwerwiegendste, denn ohne ein Wort

der Aussprache kannte ich mein

Schicksal. Die Seele spricht oft

viel beredter als man meint.

Eine schwere Nacht, viele bange

Stunden folgten. Da standen

meine Mädchenjahre vor mir,

schön $\mathrm{u}$. Lust umflossen wie

Themen in privaten Briefen gemeint. Vgl. zu Briefmustern und Subgattungen des Liebesbriefs Wyss 2010 u. 2015 sowie Wich-Reif 2012, 599.

27 Vgl. Lehmkuhl 2014, 19. 
ein Traum; - dort ein Tor wo ich hineinsehen durfte in das Leben - Lange schaute ich mir das Bild an. Langsam nahm ich Abschied von meiner ungebundenen Jugendzeit, ein großes, neues Leben begann. Als der Morgen tagte war ich bereit dir zu folgen, wenn du mit diesem Wunsch an mich herantreten würdest. So war es mein lieber Rudolf, erst hab ich dir prüfend, dann liebend $u$. vertrauend ins Auge geschaut und nun bin ich stark u. mutig genug mit dir das große Leben aufzunehmen. Gott wird uns den Weg zeigen. So wie Licht $u$. Schatten in der Landschaft wechseln, so wird auch unsere Zukunft wechselnde Stimmungen bergen. - Ich muß lachen, jetzt denkst Du: „Abendstimmung.“ - Nein schau, es ist mein tiefster Wunsch daß du von mir ein richtiges Bild im Herzen trägst. Die ernsten Seiten des Lebens stehen mir immer so klar vor Augen, ich will sie sehen, damit ich keinen Sonnenstrahl entfliehen lassen den mir Gott gütig sendet. Ich vergleiche das Leben mit einem Gewebe, wir spinnen graue u goldene Fäden hinein. Ich will kein graues Tuch, drum fange ich den Sonnenschein, damit die goldenen Strahlen leuchtend drüber stehn. - Laß mich noch ein bisschen weiter so plaudern. Oder legst du den Brief beiseite? Hast du nicht gern, wenn ich tiefer greife? Doch ja, es wird dich nicht fremd berühren. Du selbst sagtest mir, daß du über nichts leicht hinweg gehen könntest. - Mein Herz hat immer einen kleinen Kummer. Wenn ich mich dir jetzt schon so ganz gebe, wenn alles was ich habe auch dir gehört, wie soll das werden, wenn Deine Eltern einmal so gar nicht; einfach nicht einverstanden wären. Schau das ist der dunkle Punkt, der sich immer in mein sonniges Glück schleicht. Ich muß noch einmal mit dir darüber sprechen, du mußt das begreifen. Ich zweifle keinen Augenblick, daß deine Liebe zu mir groß u stark ist; aber wirst Du stark genug sein allen Anstürmen, die man auf dich macht, stand zu halten? - Wirst Du siegen? - Ich hoffe u. baue darauf. Gründe, besonders Gründe, die mich auf die Gedanken bringen weiß ich keine aufzuweisen, ich weiß nur, daß sich die Menschen allem Neuen erst feindlich entgegen setzten. Ich bin gern bereit mit dir auch diese dunkeln Stunden durchzumachen, nur siege! - Jetzt komme ich endlich zu deinem lieben Brief. Ich habe heute den ganzen Tag mit Ungeduld darauf gewartet. Ich fühlte erst ob es drin sei. Deine neuen Pläne habe ich mit großer Spannung gelesen. Was so weit weg willst du von mir? Das sind ja sicher zwei Tage zureisen. Und so lang muß ich warten, bis ich dir wieder schreiben darf. Jeden Abend meine ich ja ich müßt mich hinsetzen u. mit Dir plaudern das gibt ein großes, langes Entbehren, das erste - Aber es ist ein Schritt vorwärts! Ich laß Dich in den Norden ziehen, ich werde nur mit Post u. Eisenbahn gut Freundschaft halten. Am nächsten Freitag liegt ein 
langer Brief für dich in

Hanover, zum ersten Mal in

deiner Eltern Haus. Ich meine

er könnte zu viel sprechen.

Ich muss ihm das vor der Reise

noch tüchtig einprägen, daß er

ja nicht Lust bekommt mit deinem

Vater Freundschaft zu schließen;

ich werde das persönlich

besorgen. Hoffentlich kommt

dieser Brief noch zur rechten

Zeit in Ludwigsburg an.

$\mathrm{Zu}$ welcher Schwester reist du jetzt? Wenn der Fall bei deinem Schwager nur keine weitern

Folgen hat. - Jetzt weiß ich

dann nicht mehr, wo ich dich suchen

muß, die Gegenden kenne ich

gar nicht. Kannst du mir doch

manchmal schreiben? Glaubst

$\mathrm{Du}, \mathrm{da}$ dir eins von den

Gütern passen wird? Ich freue

mich, daß du zu Haus einen

Besuch machst. Die Mutteraugen

werden dich prüfend

ansehen. Was macht dein Appetit?

Hier in Ermatingen ist es unheimlich;

dein $u$. mein Name werden in jedem Haus ge-

nannt.
Anny kommt immer mit

neuen Berichten heim, sie fragen

sie aus was sie können. Wir

waren am Ende doch ein wenig

unvorsichtig. Vater ist es unbehaglich,

da doch noch gar nichts

offiziell ist. Wer wohl die Geschichte

so herum getragen hat? Denk

dir Marga war heute beim Zahnarzt

u. der hätte sie gefragt, wie es mir

in meiner Brautzeit ginge.

Das ist doch unheimlich. - Hille

ist heute zweimal am Haus

vorbei gefahren. Hast mit dem

Hard noch Verbindung? Dr. P.

ist jetzt in Basel, er möchte

meinen Vater kennen lernen.

Frau Schellenberg werde ich die Grüße

ausrichten. Lebwohl mein

lieber, lieber Rudolf

sei umarmt $\mathrm{u}$ fest geküßt

von deiner treuen

Emmy.

seitlich:

Von wegen dem Küssen ist es nicht so, ich habe mich geändert,

wenn Du hier wärest, würdest Du zwanzig bekommen. ${ }^{28}$

Eine Verlobungskorrespondenz dient zwar zur Eheeinleitung, doch werden mit dem einzelnen Schreiben über den kommunikativen Austausch hinweg soziale Identitäten, Rollen und Situationswissen hinsichtlich des Verlobungsnetzwerks ausgetauscht, geklärt und gesichert.

Dabei wird deutlich, dass neben der Festigung und Stabilisierung des bereits präexistierenden persönlichen, familialen und gesellschaftlichen Netzwerks ein gewisses Risiko bleibt und das Netzwerk noch als fragil wahrgenommen wird.

Wenn bei diesem Schreiben ein Liebesbrief vorliegt, der innerhalb einer Verlobungskorrespondenz geschrieben wird, kann damit nicht zum Ausdruck gebracht werden, in welcher spezifischen Situation sich die Bildung des Netzwerks befindet, hier das Netzwerk um die beiden Verlobten, das sich durch weitere Briefe und in

28 Liebesbriefarchiv Koblenz. Sign. LB_0088_0002. 
weiteren klärenden Unterredungen, Verhandlungen und Gesprächen zwischen Akteur^innen zu einem stabilisierten Netzwerk herausbilden dürfte.

Der Brief unterscheidet als Akteur`innen im Verlobungsnetzwerk verschiedene Ebenen der Netzwerkbildung: von der paarseitigen zur familialen, dann weiter zur dörflichen Gesellschaft. Es treten in dem Schreiben zudem weitere Persönlichkeiten auf, die durch ihre Beziehung zum Paar, zu Emmys Familie bestimmte, hier nicht explizierte Positionen einnehmen, die dem Bräutigam allerdings (dennoch) im Vertrauen kommuniziert werden und damit das Band zwischen Emmy und Rudolf stärken.

Insbesondere zeigt sich ein beachtlicher Kontrast zwischen Emmys Vater, der eine ihm wichtige Unterscheidung zwischen einem offiziellen und nicht offiziellen Verlöbnis vornimmt, die die Dorfbevölkerung aus welchen Gründen auch immer nicht in gleicher Weise vorzunehmen scheint.

So vermittelt der Brief nicht einzig Emmys Wissen und Position, sondern darüber hinaus die Erwartungen des Vaters, der Schwester und der Dorfbevölkerung, durch die auch Emmys Situation und ihr aktueller lebensweltlicher Kontext kommunizierbar werden. Es erscheint so, als ob sie an ihrem Vater und an der Dorfbevölkerung ihrem Bräutigam die Ernsthaftigkeit ihrer Situation darstellen könnte.

Aus Emmys Sicht kann sie dieses Wissen mit ihm teilen, weil sie davon ausgeht, dass er kognitiv und emotional in der Lage ist, diese Situation nachzuvollziehen.

Der Brief veranschaulicht damit gerade nicht eine Intentionalität der Autorin, sondern führt hier als Akteur die lebensweltliche Dynamik und die mehrstimmig durchdrungene Anlage des noch nicht abgestimmten komplexen VerlobungsNetzwerks im Geflecht zwischen sozialen Normen und der Einübung der Rollen der Braut und des Bräutigams vor. Der Brief leistet daher einen zentralen Beitrag für die Aushandlung des weiteren Verlobungs-,geschäfts', das sich nicht einzig zwischen Emmy und Rudolf entfaltet, sondern auch weitere Prozesse der Konvergenzbildung in mehreren Radien im Umkreis des Paares entstehen lässt, die sich um die Familien herum, wie auch um die Dorfgemeinschaften als Verästelungen des weiteren Netzwerks formieren.

\subsection{Beispiel 2: Liebes-Postings in sozialen Medien (am Beispiel von Facebook)}

Eine wichtige Form der Netzwerkbildung um Liebespaare findet heutzutage in sozialen Medien statt. Die sozialen Medien gelten bisweilen sogar als eine prototypische Art der Netzwerkbildung. So werden auf Plattformen wie Facebook, Insta- 
gram oder Snapchat Paare häufig als vernetzte Facebook-Identitäten ${ }^{29}$ (-Profile) präsentiert, denen nähere und entferntere Freund ${ }^{\star}$ innen auf der Plattform, folgen'. Auf diesen Plattformen entstehen Netzwerke, die sich in einer Verzahnung von sprachlicher Praxis mit plattformenspezifischen Technologien herausbilden..$^{30}$

Die eigentliche liebesbriefähnliche Kommunikation geschieht dann - einmal eher textbetont oder aber (meist) bildbetont - als Posting auf der persönlichen Pinnwand (Story) als ein direktes öffentliches Anschreiben an den Partner, in welchem im ersten Beispiel (siehe unten) in flüchtiger und spontaner Art eine Liebeserklärung zum Ausdruck gebracht wird, um gerade zu fragen, weswegen er denn nicht vorbeigekommen sei. Dies wird je nach den gesetzten Öffentlichkeitsparametern bei Facebook einem kleineren, mittleren oder größeren Freundes- bzw. Bekanntenkreis zugänglich gemacht. ${ }^{31}$ Postings sind dabei die „zentrale Produktionsund Distributionspraktik digitaler Kommunikation“32 in den sozialen Medien. Die Online-Kommunikate sind multimodal, sie bestehen aus visuell gestalteten (Ober-)Flächen, in die textuelle Elemente und Bildmaterialien zu stehen kommen. Darüber hinaus verfügen sie über weitere internettypische Elemente, so zum Beispiel Metadaten, Buttons oder Icons, die internettypische Mikropraktiken wie das Sharen, Liken, Replying auslösen. ${ }^{33}$

Es ist davon auszugehen, dass die Paare bereits als solche bekannt sind, also unumstritten als Paar auftreten und betrachtet werden. Durch den kommunikativen Akt des Postings erfolgt eine Überschreibung dieses Status in die digitale Sphäre der Facebook-Öffentlichkeit, die durch positive Kommentare von den ,Freunden“

29 Ferner können auf Plattformen wie Facebook neben Einzelprofilen auch kumulierte Paarprofile eingerichtet werden, wodurch eigentliche Paaridentitäten gebildet werden, vgl. Wyss 2017.

30 Auf den Plattformen Snapchat und Instagram hat sich eine stark auf das Bild fokussierte Liebesund Paarkommunikation herausgebildet. Bei Twitter hingegen bleiben öffentliche LiebesbriefTweets eine Ausnahme. Von Bedeutung sind sie vor allem bei prominenten Paaren.

Die Anerkennung des Paares ist nicht nötig, vielmehr zeigt sich eine Anteilnahme, eine positive oder negative Kommentierung von persönlichen Erlebnissen, Entscheidungen, die für Paarbildungsprozesse dennoch wichtig sein können. Die Angabe des Beziehungsstatus (auf Facebook), einer Information, die es einem Paar erlaubt die Beziehungsart in verschiedenen Statuskategorien zu differenzieren, bildet durch die Notwendigkeit der kommunikativen Aushandlung in der Regel auch eine Zäsur in der privaten Beziehungsdefinition.

31 Das Netzwerk gestaltet ein Profil eines/einer jeden Einzelnen durch eine gewisse technologische Anlage. Die Beiträge (Postings) werden als Text oder als Bild-Text-Ensemble direkt auf der eigenen Facebook-Seite gepostet, wobei gewählt werden kann, in welchem Kreis (der ,Freunde‘) der jeweilige Beitrag gelesen werden kann. Auf ein Posting folgt in der Regel ein Kommentar, meist eine positive Evaluierung, durch einzelne ,Freunde‘, auf die der/die Autor`in mit einem kleinen Dank (oder andersartiger Anschlusskommunikation) reagiert (vgl. Bittner 2019, 21).

32 Dang-Anh 2019, 110.

33 Vgl. Marx u. Weidacher 2019, 66-78; Dang-Anh 2019, 111-112. 
angezeigt und damit auch bestätigt wird. Das enrolment kann allerdings durch weitere kommunikative Modalitäten erfolgen, z.B. ist die bildliche Kommunikation beliebt, etwa mit Fotos, Icons und Emojis, wie es in Abb. 2 geschieht. $\mathrm{Zu}$ einem auführlichen Austausch über das Netzwerk kommt es hier nicht.

Allerdings ist die Intimität, die sich in dem ersten spontanen LiebesbriefPosting zeigt, verblüffend. Erstaunlicherweise erfolgen auch hier keine verschämten, sondern standardisierte Reaktionen in sprachlichen Kommentaren oder auch Likes. (Wenn auch ein Like bevorzugt wird und eine sprachlich formulierte Antwort auf einen Kommentar selten ist, so liest man in Facebook nicht selten auch knappe Dankesworte, die die Netzwerkbildung bestätigen.) Damit tritt der technische Aspekt des Kommunizierens in den Vordergrund und der Eindruck, dass die vielen Aktivitäten allein der Bekräftigung der technologischen Netzwerkstruktur dienen, scheint offensichtlich.

Obschon in der Vorstrukturiertheit des funktionalen Gefüges der Plattform Facebook eine gewisse Transparenz entsteht, geschieht die Netzwerkbildung reaktiv, relativ spontan, wenn nicht sogar automatisiert. Eine deliberative und intentionale Bildung des Netzwerks, wie es in Abb.1 erfolgt, ist hier nicht in gleicher Art vorgesehen.

Gleichzeitig gibt es eine deutliche interaktionale Anlage, die ein spontanes, knappes Posting oder einen Kommentar in Reaktion auf die Postings möglich macht. Der Spontaneität geschuldet ist jedoch die Verwendung informeller Schriftlichkeit, ${ }^{34}$ die zu bestimmten Anlässen in kleinschrittiger Kommunikationsweise durch die Folgen von Posting - Kommentar - Like sozusagen in kleinen Häppchen geschieht.

Zudem wird bei einer Veröffentlichung auf Facebook ein Druck generiert, da die anteilnehmende Öffentlichkeit - das inhärente Netzwerk - auf den ,nächsten Schritt' wartet. Auch dies führt zu schnellen, spontanen, vielfach stereotypen Reaktionen, die mehr und mehr standardisiert werden, wenn sie nicht bereits standardisiert oder in Icons vorliegen.

Doch gibt es einen Ansporn und Anspruch durch das Ranking, das die Postings voneinander abhebt. So fragt es sich dabei stets auch, wie groß der (Facebook-)Freundeskreis ist und wie stark sich diese Freunde an der persönlichen Liebeskommunikation beteiligen. ${ }^{35}$

34 Obschon die Normentoleranz in Online-Postings üblich ist, lässt es sich nicht immer exakt entscheiden, in welchen Fällen dieser Informalität - wie Imo (2012) dies unterscheidet - eine Schreibhaltung oder auch ein gewisser Kompetenzverlust zu Grunde liegt.

35 Vgl. Bittner 2019, 40. 


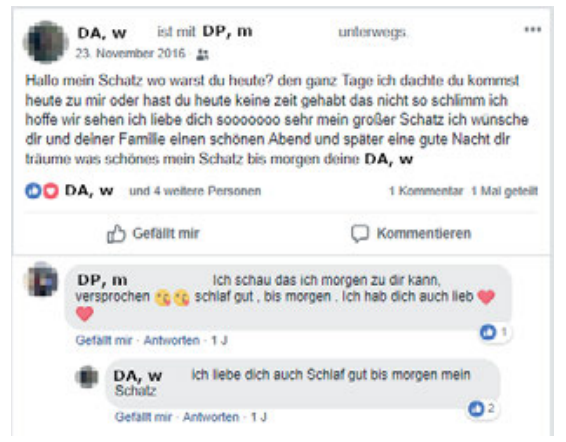

Abb. 1: Textbetontes liebesbriefartiges Posting.

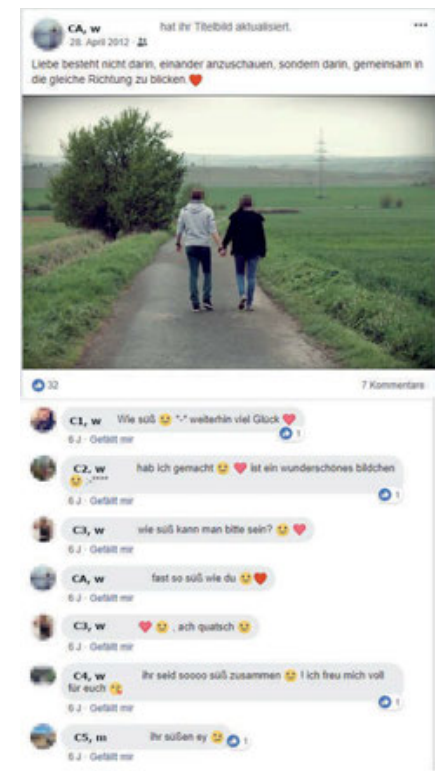

Abb. 2: Bildbetontes Posting.

So wird auch bei Facebook ein Netzwerk um ein Paar geschaffen, das durch seine mehrschichtige Strukturierung technologisch komplexer ist in seiner Anlage, und über eine Vielzahl von Akteur^innen verfügt, die nebeneinander und gleichzeitig tätig sein können. Es ist daher umso schwieriger die Netzwerkbildung nachzuvollziehen, da die Zulassung als Freund ${ }^{\star}$ in zum Netzwerk und die Ausformung sozialer Normen in der Netzwerkkommunikation einerseits einem Druck unterliegt und zugleich auch einem Wandel unterworfen ist. ${ }^{36}$

Da in diesen technologisch vorgefertigten Netzwerken eine Vielzahl von einander mehr oder weniger bekannten Akteur`innen beteiligt sind, die oft gerade nicht zum engeren familiären oder freundschaftlichen Kreis gehören (müssen), bilden sich hier supplementartige Netzwerke, die formal durch die bei Facebook etablierten Mikropraktiken des Kommentierens, Likens, Teilens, in ihrer Wirkung als Akteure dazu beitragen, das Netzwerk zu bilden.

36 Bittner (2019) berichtet in seiner Studie, dass die Paare ihre wichtigen Netzwerkpartner^innen kaum über das technologische Netzwerk kontaktieren, sondern einen inneren Kreis der nahe stehenden Personen meist mündlich oder über andere Medien der ,Nähe‘ wie Messenger oder das Telefon über wichtige Entscheidungen informieren oder zu bedeutenden Fragen kontaktieren. Dies bedeutet nichts anderes, als dass neben der technologischen Verzahnung die Bildung von analogen Netzwerken für die Netzwerkbildung von Liebespaaren wichtig ist. 
Dabei werden Praktiken des enrolment, die üblicherweise sprachliche und soziale Aushandlungen erfordern würden, durch technologisch standardisierte Verfahren ,ersetzt', wodurch diese wichtigen Momente, die zu Konvergenz und Irreversibilität führen könnten, im Dunkeln bleiben.

\section{Fazit}

Weder die älteren intimen Liebesbriefe noch Postings sind einzig für den amourösen Austausch zwischen Liebespaaren gedacht. Gemeinsam ist ihnen eine vernetzende Funktion, die Funktion der Netzwerkbildung um das Liebespaar, wenn auch in unterschiedlicher Art.

Bereits im 19. Jahrhundert erfolgt durch die enge Bindung von Braut und Bräutigam an die Familie und durch die Praxis des Vorlesens eine innerfamiliäre Vernetzung, bei der noch unverheiratete Paare eine Art hypothetischen Nukleus bilden. Ähnlich geschieht es durch die vernetzte Öffentlichkeit in sozialen Medien, in welchen Paare intime Kommunikation austauschen.

Mit der Akteur-Netzwerk-Theorie wurde die über 100 Jahre auseinanderliegende Netzwerkbildung verglichen und es wurden wesentliche Unterschiede gezeigt.

Bei der Verlobungskorrespondenz bildet sich ein komplexes enrolment aus, bei dem verschiedene Akteure in einem Brief zur Sprache kommen. Die Mehrstimmigkeit dieser Netzwerkbildung weist auf die komplexe soziale Verzahnung emotionaler und sozialer Ebenen hin, welche die Akteure berücksichtigen, indem sie Vermittlungsangebote, Vorübersetzungen anbieten, durch die Bedeutungen ko-konstruiert werden.

Bei den Postings geschieht die Bedeutungs-ko-konstruktion in der Regel durch relativ stark standardisierte Kommunikationsverfahren. Davon profitieren die durch die Beschleunigung und Spontaneität eher als reaktiv erfahrenen Beiträge.

Briefe und Postings wurden auch hinsichtlich der Netzwerkbildung neu bewertet und können damit in ihrer sozialanthropologischen Bedeutung neu beleuchtet werden. Der Vorteil eines solch (radikal) konstruktivistischen Zugangs ist die methodische Umkehrung auf das ,Wie“ der Netzwerkbildung durch den Brief und damit ein Blick auf den Brief hinsichtlich seiner technischen und sozialen Bedingungen, seiner Materialität als Briefobjekt und seiner Medialität als Netzwerkakteur zugleich. Dabei wird die materialitätstheoretische Dimension des Briefes (und des Postings) auf einer weiteren Ebene deutlich gemacht: Der Brief (und das Posting) als ein Netzwerk-Akteur im Verlobungs- und Paarnetzwerk ,in progress‘. 


\section{Literatur}

Arnim, Achim von u. Bettine Brentano, verh. von Arnim (2018). Briefwechsel. Hg. v. Renate Moering. Wiesbaden.

Bittner, Sebastian (2019). ANT goes Facebook. Eine Untersuchung von Liebesnetzwerken auf Facebook mit Hilfe der Akteur-Netzwerk-Theorie. Bachelorarbeit Universität Koblenz. Ref.: Eva Lia Wyss. Koblenz. (unveröffentlicht), Liebesbriefarchiv (LBA, liebesbriefarchiv.de) an der Universität Koblenz.

Bohnenkamp, Anne u. Waltraud Wiethölter (Hg.) (2008). Der Brief-Ereignis \& Objekt. Frankfurt. Callon, Michel (1986). „Some Elements of a Sociology of Translation: Domestication of the Scallops and the Fishermen of St Brieuc Bay“, in: Power, Action and Belief. A New Sociology of Knowledge? Hg. v. John Law. London: 196-232.

Callon, Michel (1991). „Techno-Economic Networks and Irreversibility“, in: A Sociology of Monsters. Essays on Power, Technology and Domination, Sociological Review Monograph 38. Ed. by John Law. London: 132-161.

Castells, Manuel (2000). The Rise of the Network Society. Oxford.

Castells, Manuel (2004). „Informationalism, Networks, and the Network Society: A Theoretical Blueprint“, in: The Network Society: a cross-cultural perspective. Hg. v. dems. Cheltenham: 3-45.

Dang-Anh, Mark (2019). Protest twittern. Eine medienlinguistische Untersuchung von Straßenprotesten. Bielefeld.

Ehlich, Konrad (2014). „Eine kurze Pragmatik des Briefs“, in: Fontanes Briefe ediert. Hg. v. Hanna Delf von Wolzogen u. Rainer Falk. Würzburg: 14-35.

Ermert, Karl (1979). Briefsorten. Untersuchungen zur Theorie und Empirie der Textklassifikation. Tübingen.

Frick, Karina (2014). „Liebeskommunikation über Facebook. Eine korpusbasierte Untersuchung kommunikationstheoretischer und sprachlicher Merkmale der Paar-Kommunikation auf Facebook“, in: Networx 65. https://www.mediensprache.net/de/networx/networx-65.aspx.(10. 2.2020).

Hausendorf, Heiko (2009). „Die Zuschrift. Exemplarische Überlegungen zur Methodologie der linguistischen Textsortenbeschreibung“, in: Zeitschrift für Sprachwissenschaft 19,2: 210-244.

Hübener, Andrea, Jörg Paulus u. Renate Stauf (2020). „Liebesbrief/Erotischer Brief“, in: Handbuch Brief. Von der Frühen Neuzeit bis zur Gegenwart. Hg. v. Marie Isabel Matthews-Schlinzig, Jörg Schuster, Gesa Steinbrink u. Jochen Strobel. Bd. 1: Interdisziplinarität - Systematische Perspektiven - Briefgenres. Berlin u. Boston: 505-514.

Imo, Wolfgang (2012). Informelles Schreiben als Indikator für Sprachverfall? Normabweichungen in Produktbeschreibungen privater Verkäufer in einem Online-Auktionshaus. Berlin.

Jansen, Dorothea (2003). Einführung in die Netzwerkanalyse. Opladen.

Johnson, Jim (1988). „Mixing Humans and Nonhumans Together. The Sociology of a Door-Closer“, in: Social Problems 35,3: 298-310.

Lach, Roman (2020). Der Brautbrief, in: Handbuch Brief. Von der Frühen Neuzeit bis zur Gegenwart. Hg. v. Marie Isabel Matthews-Schlinzig, Jörg Schuster, Gesa Steinbrink u. Jochen Strobel. Bd. 1: Interdisziplinarität - Systematische Perspektiven - Briefgenres Berlin u. Boston: $515-523$. 
Latour, Bruno (1992). „Where are the Missing Masses? The Sociology of a Few Mundane Artifacts“, in: Shaping Technology; Building Society. Studies in Sociotechnical Change. Hg. v. Wiebe E. Bijker u. John Law. Cambridge, Mass.: 225-258.

Latour, Bruno (1995). Wir sind nie modern gewesen. Versuch einer symmetrischen Anthropologie. Berlin.

Latour, Bruno (1996a). Der Berliner Schlüssel. Erkundungen eines Liebhabers der Wissenschaften. Berlin.

Latour, Bruno (1996b). „On Interobjectivity“, in: Mind, Culture, and Activity 3,4: 228-245.

Latour, Bruno (2007). Eine neue Soziologie für eine neue Gesellschaft. Eine Einführung in die Akteur-Netzwerk-Theorie. Frankfurt a. M.

Lehmkuhl, Ursula (2014). „Reading Immigrant Letters and Bridging the Micro-Macro Divide“, in: Studia Migracyjne - Przegląd Polonijny 40,1: 9-30.

Marx, Konstanze u. Georg Weidacher (2019). Internetlinguistik. Tübingen.

Nickisch, Reinhard M. G. (1991). Brief. Stuttgart.

Schulz-Schaeffer, Ingo (2014). „Akteur-Netzwerk-Theorie. Zur Ko-Konstitution von Gesellschaft, Natur und Technik“, in: Soziale Netzwerke. Hg. v. Johannes Weyer. Berlin: 267-290.

Schuster, Britt-Marie (2020): „Linguistik“, in: Handbuch Brief. Von der Frühen Neuzeit bis zur Gegenwart. Hg. v. Marie Isabel Matthews-Schlinzig, Jörg Schuster, Gesa Steinbrink u. Jochen Strobel. Bd. 1: Interdisziplinarität - Systematische Perspektiven - Briefgenres. Berlin u. Boston: 19-39.

Stauf, Renate u. Jörg Paulus (Hg.) (2013). SchreibLust: Der Liebesbriefim 18. und 19. Jahrhundert. Berlin u. Boston.

Strobel, Jochen (2020). „Der Brief als Gabe“, in: Handbuch Brief. Von der Frühen Neuzeit bis zur Gegenwart. Hg. v. Marie Isabel Matthews-Schlinzig, Jörg Schuster, Gesa Steinbrink u. Jochen Strobel. Bd. 1: Interdisziplinarität - Systematische Perspektiven - Briefgenres. Berlin u. Boston: 254-268.

Weber, Max (1922). Wirtschaft und Gesellschaft. Grundriss einer verstehenden Soziologie. Tübingen. https://www.textlog.de/7319.html [Zugriff: 10.10.2020].

Wich-Reif, Claudia (2012). ,....obwohl ich heute [...] mich früh hierher begab, um brieflich mit Dir zu plaudern'. Die Syntax des ,Plauderns“ in Briefen des 19. Jahrhunderts“, in: Syntaktischer Wandel in Gegenwart und Geschichte. Akten des Kolloquiums in Montpellier vom 9. bis 11.6.2011. Hg. v. Michel Lefèvre. Berlin: 598-614.

Wyss, Eva (2008). „From the Bridal Letter to Online Flirting: Changes in Text Type and Writing Practice from the 19th Century to the Internet Era“, in: Journal of Historical Pragmatics 9,2: 225-254.

Wyss, Eva (2010a). „Verlobungskorrespondenzen, Brautbriefe und Online-Flirts. Schriftliche Liebeskommunikation vom 19. Jahrhundert bis in die Internet-Ära“, in: Muster und Variation in Medientexten. Hg. v. Martin Luginbühl u. Daniel Perrin. Bern: 81-123.

Wyss, Eva (2010b) „Der Liebesbrief zwischen Kunst, Alltagsschriftlichkeit und populärer Kultur. Zur Bestimmung und historischen Variation einer Textsorte,“ in: „Und wer bist du, der mich betrachtet?" Populäre Literatur und Kultur als ästhetische Phänomene. Hg. v. Helga Arend. Bielefeld: 351-373.

Wyss, Eva (2017). vermiss dich krass my love. Schweizer Liebeserklärungen 2.0. Zürich. 
